\title{
Scanning Electron Microscopy and Spectroscopy Characterization of Cobalt Ferrite Nanoparticles by a Facile Hydrothermal Synthesis Method
}

\author{
Jessica Vinh, Xinlin Liu, Ruben Contreras, James Barnes, Andrea Goforth and Jun Jiao
}

Portland State University, Portland, Oregon, United States

Cobalt ferrite $\left(\mathrm{CoFe}_{2} \mathrm{O}_{4}\right)$ has been attracting great attention due to its importance in magnetic and electronic materials industries. Its properties make it widely applicable and sought after in several fields, such as magneto-optics and energy storage ${ }^{[1]}$. This study aims to develop a synthesis method that uses an environmentally friendly solvent in the hydrothermal process for the formation of cobalt ferrite nanoparticles.

To synthesize $\mathrm{CoFe}_{2} \mathrm{O}_{4}$ nanoparticles, the solvothermal method is a preferred choice due to its facile procedures $^{[1][2][3]}$. A 2:1 stoichiometric ratio of iron acetylacetonate and cobalt acetylacetonate reactants were stirred in $20 \mathrm{~mL}$ of deionized (DI) water for 24 hours, and then transferred to a stainless-steel autoclave and heated for 48 hours at a temperature of $180^{\circ} \mathrm{C}$. The nanoparticles were washed five times with DI water and dried in a vacuum oven for 5 hours. Scanning electron microscopy (SEM), energy dispersive x-ray spectroscopy (EDS), x-ray diffraction (XRD), and Raman spectroscopy were used for characterization.

The microscopy and spectroscopy techniques mentioned above play indispensable roles in allowing systematic characterization of the samples and enabling optimization of the synthesis processes. The morphology and sizes of the samples were investigated by SEM. From figure 1 (a) and (b), it can be seen that the average size of the $\mathrm{CoFe}_{2} \mathrm{O}_{4}$ nanoparticles was in the range of 30-60 nm and the particle surface was clean and smooth. It can also be observed that the shapes of the nanoparticles are polyhedral, indicative of crystalline morphologies. The larger conglomerates of the nanoparticles appear to be cubic in shape. The resulting nanoparticle size is larger than the size range prescribed in most literature corresponding to experiments that use different solvents. As an example; studies using ethanol as a solvent had nanoparticles around $\sim 20 \mathrm{~nm}$ in diameter ${ }^{[3]}$, while another study using acetophenone as a solvent resulted in $\sim 11 \mathrm{~nm}$ nanoparticles ${ }^{[1]}$. The reaction time chosen is also longer than all referenced papers mentioned in both mixing time and time in the oven. The EDX chemical analysis of the samples was also performed. Figure 1 (d) shows an EDX spectrum of the area indicated in figure 1 (c). The result confirms the existence of the elements $\mathrm{Co}, \mathrm{Fe}, \mathrm{O}$, which are the key components of $\mathrm{CoFe}_{2} \mathrm{O}_{4}$. The Si signals in the spectrum are from the substrate and the carbon signals are due to sample contamination. The phase information and crystal structure of the samples were examined by XRD. As shown in figure 2 (a), a single cubic spinel phase $(\mathrm{Fd}-3 \mathrm{~m}$ with lattice parameter $\mathrm{a}=8.38 \AA$ ) showed no additional reflections, thus indicating phase purity of the material. The patterns were matched to the Crystallography Open Database (COD) card \#1533163 using pattern matching software (PDXL2 version 2.6). The strong reflections indicate high crystallinity. The XRD patterns of $\mathrm{CoFe}_{2} \mathrm{O}_{4}$ nanoparticles synthesized with DI water match with the observed peaks from the reports by S. H. Xiao ${ }^{[4]}$ and P. Chandramohan ${ }^{[2]}$, further confirming the nanoparticles to be spinels. Raman spectroscopy is also used to verify the formation of cobalt ferrite and the result is shown in figure 2 (b). As indicated in other literature that use Raman spectroscopy to characterize cobalt ferrite ${ }^{[2][5][6]}$, cubic spinel structures have five Raman active modes $\left(1 \mathrm{~A} 1 \mathrm{~g}, 1 \mathrm{E}_{\mathrm{g}}\right.$, and $3 \mathrm{~T}_{2 \mathrm{~g}}$ ). Note in figure 2 (b) there were four prominent Raman active modes visible. They are shown to 
have peaks at $674\left(\mathrm{~A}_{1 \mathrm{~g}}\right), 551\left(\mathrm{~T}_{2 \mathrm{~g}}(1)\right), 453\left(\mathrm{~T}_{2 \mathrm{~g}}(2)\right)$, and $306\left(\mathrm{E}_{\mathrm{g}}\right) \mathrm{cm}^{-1}$, which can be compared to $\mathrm{P}$. Chandramohan's ${ }^{[3]}$ values of $674.8\left(\mathrm{~A}_{1 \mathrm{~g}}\right), 547.7\left(\mathrm{~T}_{1 \mathrm{~g}}(1)\right), 460.7\left(\mathrm{~T}_{2 \mathrm{~g}}(2)\right), 190.2\left(\mathrm{~T}_{1 \mathrm{~g}}(3)\right)$, and $293.2\left(\mathrm{E}_{\mathrm{g}}\right)$ $\mathrm{cm}^{-1}$. The $\mathrm{T}_{2 \mathrm{~g}}(3)$ peak would be found at $\sim 190 \mathrm{~cm}^{-1}$, the location of said peak designated by dashed line in figure 2 (b). The matching software did not identify it due to the peak being low and possibly covered by the background noise of the system. The peak at $674 \mathrm{~cm}^{-1}$ shows the stretching vibration of the $\mathrm{O}^{2-}$ and $\mathrm{Fe}^{3+}$ ions in the tetrahedral site. The $\mathrm{Co}^{2+}$ ions are found in the octahedral sites, which correspond with all peaks below $600 \mathrm{~cm}^{-1}$. Figure 2 (b) experiences a red shift from P. Chandramohan and N. Jansen's Raman spectra ${ }^{[2][3]}$, which explains the absence of a secondary A1g peak that is often found, such as the peak at $604.9 \mathrm{~cm}^{-1}$ in P. Chandramohan ${ }^{[2]}$. The shift in Raman active modes are due to change in atomic radii of the metal ions, leading to migration of metal ions to different sites ${ }^{[5]}$.

In summary, the cobalt ferrite nanoparticles were synthesized by a solvothermal method. SEM image suggests that the nanoparticles are uniform whilst the EDX confirms the chemical components of the cobalt ferrite nanoparticles. XRD and Raman show that the morphologies of the nanoparticles have welldefined cubic spinel structure. Using a DI water solvent does not change uniformity of the particles compared to other solvents, although further study is ongoing to determine the effect of reaction time in relation to particle size ${ }^{[7]}$.

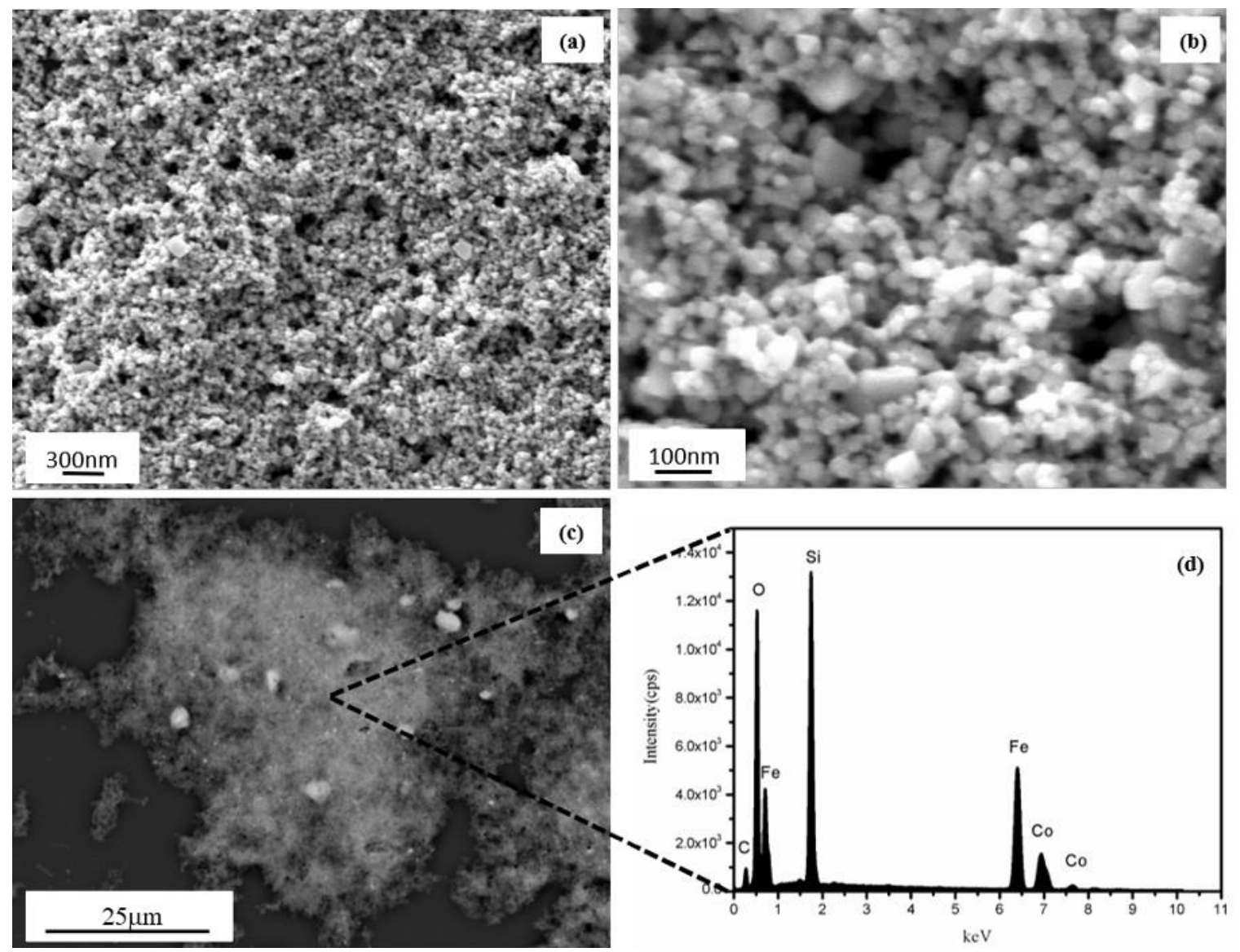

Figure 1. The SEM image of CoFe2O4 nanoparticles: (a) at a low magnification while (b) is at a relatively high magnification image. The particles are 30-60 nm in diameter. The uniformity of the nanoparticles can be seen in (a) and (b), with some larger particles seen in (b). (c) is an SEM image of CoFe2O4 
nanoparticles sample where the EDX analysis was performed. (d) shows the EDX analysis. All components of cobalt ferrite are detected in (d), the silicon peak from the substrate and the low carbon peak due to contamination.
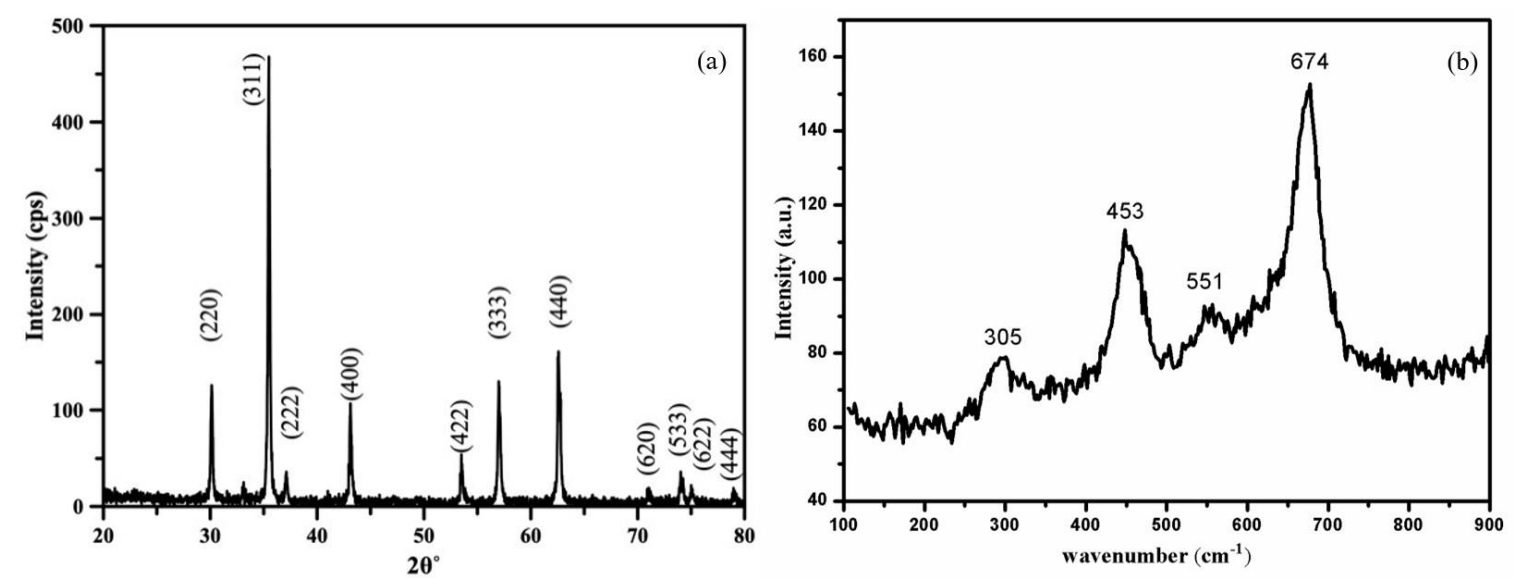

Figure 2. (a) shows the XRD pattern of cobalt ferrite nanoparticles - the peaks demonstrating high crystallinity and corresponding with cubic spinels. (b) is the Raman spectra of the nanoparticles; the active modes seen match with the active modes corresponding to the cubic spinel structure. The dashed line corresponds with the location of the $\mathrm{T} 1 \mathrm{~g}(3)$ peak, and it can be seen that there is a significant amount of background noise at that location.

\section{References}

[1] C. Vázquez-Vázquez, M. A. López-Quintela, M. C. Buján-Núñez, and J. Rivas, 13, (2010) pp. 16631676.

[2] P. Chandramohan, M. Srinivasan, S. Velmurugan, and S. Narasimhan, Journal of Solid State Chemistry, 184, (2011) pp. 89-96.

[3] N. Jansen, J. Yuzon, E. McCardle-Blunk, J. Barnes, A. Gotforth, J. Jiao, Microscopy and Microanalysis, (2019)

[4] S. H. Xiao, W. F. Jiang, L. Y. Li, and X. J. Li, Materials Chemistry and Physics, 106, (2007) pp. 8287.

[5] Z. Ding, W. Wang, Y. Zhang, F. Li, and J. Liu. Journal of Alloys and Compounds, 640, (2015) pp. 362-370.

[6] S. Naik, A. Salker, S. Yusuf, and S. Meena, Journal of Alloys and Compounds, 566, (2013) pp. 5461.

[7] The research efforts for this study is supported in part by NSF awards \#1851851 and \#1560383, and Oregon Metal Initiative. 